\title{
Interferon-Alpha-Induced Thyroid Dysfunction in Patients with Chronic Active Hepatitis C: A Transient, Reversible and Self-limited Dysfunction
}

\author{
Kumiko Tsuboi, Masahiko Katayama, Rena Yuasa, Hana Matoba, Toru Nagayama, \\ Fumie Ihara, Taeko Ooya, Kenji Matsuo, Sachio Otsuka and Yukitaka Miyachi
}

To survey the prognoses of interferon-alpha (IFN $\alpha$ )-induced thyroid dysfunction, a total of 100 patients (49 males and 51 females) with biopsy-proven chronic active hepatitis $C$ were studied. Either during or after IFN $\alpha$ therapy, 29 patients $(33.7 \%)$ revealed suppression/elevation of thyroid stimulating hormone (TSH) or both, transient thyrotoxicosis (TSH less than $0.1 \mu \mathrm{U} / \mathrm{ml}$ ) or transient hypothyroidism (TSH 5.0-190.95 $\mu \mathrm{U} / \mathrm{ml}$ ). However, the thyroid function normalized without supplementation of the thyroid hormone in the follow-up period. In the same period, one of the 14 control patients $\mathbf{( 7 . 1 \% )}$ ) developed thyroid dysfunction. Thyroid abnormalities developed significantly more in patients with IFN $\alpha$ therapy than in those without IFN $\alpha$ therapy. The findings suggest that the occult autoimmune disorder becomes overt with IFN $\alpha$ treatment in patients with pre-existent autoimmune thyroid disease. IFN $\alpha$-induced thyroid dysfunction is transient, reversible and self-limited. It is not necessary to discontinue IFN $\alpha$ therapy when thyroid dysfunction develops.

(Internal Medicine 37: 27-31, 1998)

Key words: silent thyroiditis, chronic thyroiditis, Hashimoto's thyroiditis, thyrotoxicosis, hypothyroidism, pre-existent autoimmune thyroid disease

\section{Introduction}

Interferon-alpha (IFN $\alpha$ ) is an approved drug for the treatment of chronic active hepatitis $\mathrm{C}$. Thyroid dysfunction is a well recognized side effect of treatment with IFN $\alpha(1-17)$, and some reports have advocated the discontinuance of IFN $\alpha$ therapy because of developing thyroid dysfunction in some patients. We observed 6 patients with chronic active hepatitis $\mathrm{C}$ who were diagnosed as having hypothyroidism (thyroid stimulating hormone, TSH $4.78-138.5 \mu \mathrm{U} / \mathrm{ml}$ ) after completion of IFN $\alpha$ therapy (17). Their thyroid dysfunction was transient, the clinical course was the same as that which occurs in silent thyroiditis or subacute thyroiditis. The destruction of the thyroid gland, as in silent thyroiditis, causes the release of stored thyroid hormones, inducing temporary thyrotoxicosis and subsequent hypothyroidism. In this condition, it is only necessary to treat the disease symptomatically, and most patients recover completely.

If IFN $\alpha$-induced thyroid dysfunction is transient and reversible, thyroid dysfunction should not be the prime reason to discontinue IFN $\alpha$ therapy, rather the therapy can be completed. In this prospective study, we surveyed the prognoses of IFN $\alpha$ induced thyroid dysfunction in patients with chronic active hepatitis $\mathrm{C}$.

\section{Materials and Methods}

This study was comprised of 100 patients (49 males and 51 females, aged 19 to 73 years) with biopsy proven chronic active hepatitis C. Informed consent was previously obtained from each patient, and 14 control patients were not given IFN $\alpha$ at their own request. The remaining 86 patients were randomly assigned to three groups: Group 1; 39 patients treated with natural IFN $\alpha$ (nIFN $\alpha$, Sumitomo Pharmaceutical Co., Osaka), Group 2; 19 patients treated with recombinant IFN $\alpha-2 \mathrm{a}(\mathrm{rIFN} \alpha-$ 2a, Takeda Chemical Industry Ltd., Osaka), and Group 3; 28 patients with recombinant IFN $\alpha-2 b$ (rIFN $\alpha-2 b$, Yamanouchi Pharmaceutical Co., Tokyo). The treatment regimen for IFN $\alpha$ was based on a 24-week treatment protocol of a daily intramuscular injections for the first 2 weeks, followed by 3 injections

From the First Department of Internal Medicine, Toho University School of Medicine, Tokyo

Received for publication March 27, 1997; Accepted for publication October 3, 1997

Reprint requests should be addressed to Dr. Kumiko Tsuboi, the First Department of Internal Medicine, Toho University School of Medicine, 6-11-1 Omori-nishi, Ota-ku, Tokyo 143-8541 
per week for 22 weeks; nIFN $\alpha$ was administrated in 6 mega units (MU) at a time, rIFN $\alpha-2 \mathrm{a}$ and rIFN $\alpha-2 \mathrm{~b}$ were administrated in $9 \mathrm{MU}$ at a time. The follow-up period was 6 to 14 months. Therapeutic response was assessed at 6 months after the 24-week treatment according to the serum alanine aminotransferase (ALT) concentration and liver biopsy. Response was defined as remission upon normal serum ALT concentration lasting six months after the completion of treatment.

These patients had no prior thyroid disease, lived in an iodine sufficient area, and had normal iodine diets. Thyroid studies were performed before, during and after IFN $\alpha$ therapy and during the same follow-up period for the control patients. The goiter was examined by palpation and ultrasonography (US). Fine needle aspiration biopsy (FNAB) was performed in some cases. The thyroid function was estimated by serum TSH (sensitive assay), free thyroxine $\left(\mathrm{FT}_{4}\right)$ and free triiodothyronine $\left(\mathrm{FT}_{3}\right)$ which were determined with commercially available assay kits. The normal range of each assay was as follows: TSH $0.3-4.8 \mu \mathrm{U} / \mathrm{ml}, \mathrm{FT}_{4}$ 0.8-1.9 ng/dl, $\mathrm{FT}_{3} 3.2-4.8 \mathrm{pg} / \mathrm{ml}$. We diagnosed hypothyroidism when TSH rose above $5 \mu \mathrm{U} / \mathrm{ml}$, or latent thyrotoxicosis when TSH was less than $0.1 \mu \mathrm{U} / \mathrm{ml}$, accompanied with increased or upper normal levels of $\mathrm{FT}_{4}$ and/ or $\mathrm{FT}_{3}$. The thyroid autoantibodies were measured by two methods: passive hemagglutination assay (HA, Fuji Rebio Co., Tokyo) and radioimmunoassay (RIA, RSR limited, Cardiff, UK). The thyroid microsomal antibody (MCHA) and the antithyroglobulin (Tg) antibody (TGHA) were measured by HA. The anti-thyroid peroxidase (TPO) antibody and the anti-Tg antibody measured by RIA were abbreviated to TPOAb and $\mathrm{TgAb}$, respectively. The detection limit of RIA was $0.1 \mathrm{U} / \mathrm{ml}$, and the normal range was less than $0.3 \mathrm{U} / \mathrm{ml}$. TPOAb and TgAb were determined during and after treatment.

\section{Results}

Before therapy, there were 82 patients, 40 males and 42 females, with palpable goiters including two with nodular goiters. US and FNAB revealed one nodule to be an adenoma, another an adenomatous nodule and 4 diffuse goiters as adenomatous goiters (nontoxic multinodular goiters). Small and hard elastic goiters were observed in 27 male and 27 female patients. There was no correlation between age and incidence of goiter. There were no patients with severe hypothyroidism but seven patients ( 2 males and 5 females) had subclinical hypothyroidism (TSH 5.0-19.3 $\mu \mathrm{U} / \mathrm{ml}$ ) (Table 1). For MCHA and TGHA, eight patients ( 1 male and 7 females) had either or both positive. Three euthyroid patients had positive TGHA and MCHA. Two patients with hypothyroidism and three euthyroid patients had negative TGHA and positive MCHA.

During and after IFN $\alpha$ therapy, 29 out of 86 treated patients (33.7\%) revealed TSH suppression and/or elevation or both, while one female out of 14 control patients $(7.1 \%)$ had transient elevation of TSH during the same follow-up period (Table 1). Without consideration to gender, the incidence of TSH changes did not statistically different among the three groups with chisquare analysis. Two patients revealed overt hypothyroidism, 27 patients revealed subclinical dysthyroidism. Figure 1 shows the clinical course in a 50-year-old female treated with nIFN $\alpha$, who developed hypothyroidism. The patient had a small and elastic hard goiter, and was euthyroid and had negative MCHA and TGHA before the therapy. After 20 weeks of treatment, TSH rose to $171 \mu \mathrm{U} / \mathrm{ml}, \mathrm{FT}_{4}$ decreased to $0.21 \mathrm{ng} / \mathrm{dl}$ and MCHA titer was 400 . IFN $\alpha$ therapy was continued and completed without supplementation of the thyroid hormone at her own request. Maximum TSH level was $190.9 \mu \mathrm{U} / \mathrm{ml}$ at 22 weeks, followed by a rapid reduction of TSH and an increase of $\mathrm{FT}_{4}$ during IFN $\alpha$ therapy. $\mathrm{FT}_{4}$ became normal at 6 weeks after the completion of IFN $\alpha$ therapy. The symptoms and signs of thyrotoxicosis were subtle, and the patient tolerated hypothyroidism well without supplementation of L-thyroxine. The $\mathrm{TgAb}$ and TPOAb transiently rose, and the changes in size and echo texture of the goiter were also transient. Figure 2 shows the case of a 42-year-old female treated with rIFNo-2b, who developed thyrotoxicosis. The patient had a medium-sized soft goiter, was euthyroid and had negative TGHA and MCHA before the therapy. After 16 weeks of treatment, the patient was clinically euthyroid with normal $\mathrm{FT}_{4}$, but serum $\mathrm{TSH}$ was suppressed. Two weeks later, $\mathrm{FT}_{4}$ was elevated to $3.02 \mathrm{ng} / \mathrm{dl}$.

Table 1. Thyroid Abnormalities in TSH and Antibodies before, during or after IFN $\alpha$ Therapy in Patients with Chronic Active Hepatitis C

\begin{tabular}{|c|c|c|c|c|c|c|c|c|c|}
\hline \multirow[t]{2}{*}{ Drug } & \multicolumn{3}{|c|}{ No. of patients } & \multicolumn{3}{|c|}{$\begin{array}{l}\text { No. (\%) of patients who had } \\
\text { suppression/elevation of TSH }\end{array}$} & \multicolumn{3}{|c|}{$\begin{array}{l}\text { No. of patients who had } \\
\text { positive MCHA/TGHA }\end{array}$} \\
\hline & & Male & Female & & Male & Female & & Male & Female \\
\hline before IFN $\alpha$ & 100 & 51 & 49 & $7(7.0)$ & $2(4.0)$ & $5(10.2)$ & 8 & 1 & 7 \\
\hline without IFN $\alpha$ & 14 & 3 & 10 & $1(7.1)$ & $0(0.0)$ & $1(10.0)$ & 2 & 1 & 1 \\
\hline \multicolumn{10}{|l|}{ with } \\
\hline $\mathrm{n}-\mathrm{IFN} \alpha$ & 39 & 19 & 20 & $12(30.8)$ & $4(21.1)$ & $8(40.0)$ & 5 & 1 & 4 \\
\hline $\mathrm{r}-\mathrm{IFN} \alpha-2 \mathrm{a}$ & 19 & 12 & 7 & $8(42.1)$ & $2(16.7)$ & $6(85.7)$ & 3 & 0 & 3 \\
\hline$r-I F N \alpha-2 b$ & 28 & 16 & 12 & $9(32.4)$ & $4(25.0)$ & $5(41.7)$ & 2 & 0 & 2 \\
\hline subtotal & 86 & 47 & 39 & $29(33.7)$ & $10(21.3)$ & $19(48.7)$ & 10 & 1 & 9 \\
\hline
\end{tabular}


IFN-Induced Thyroid Dysfunction

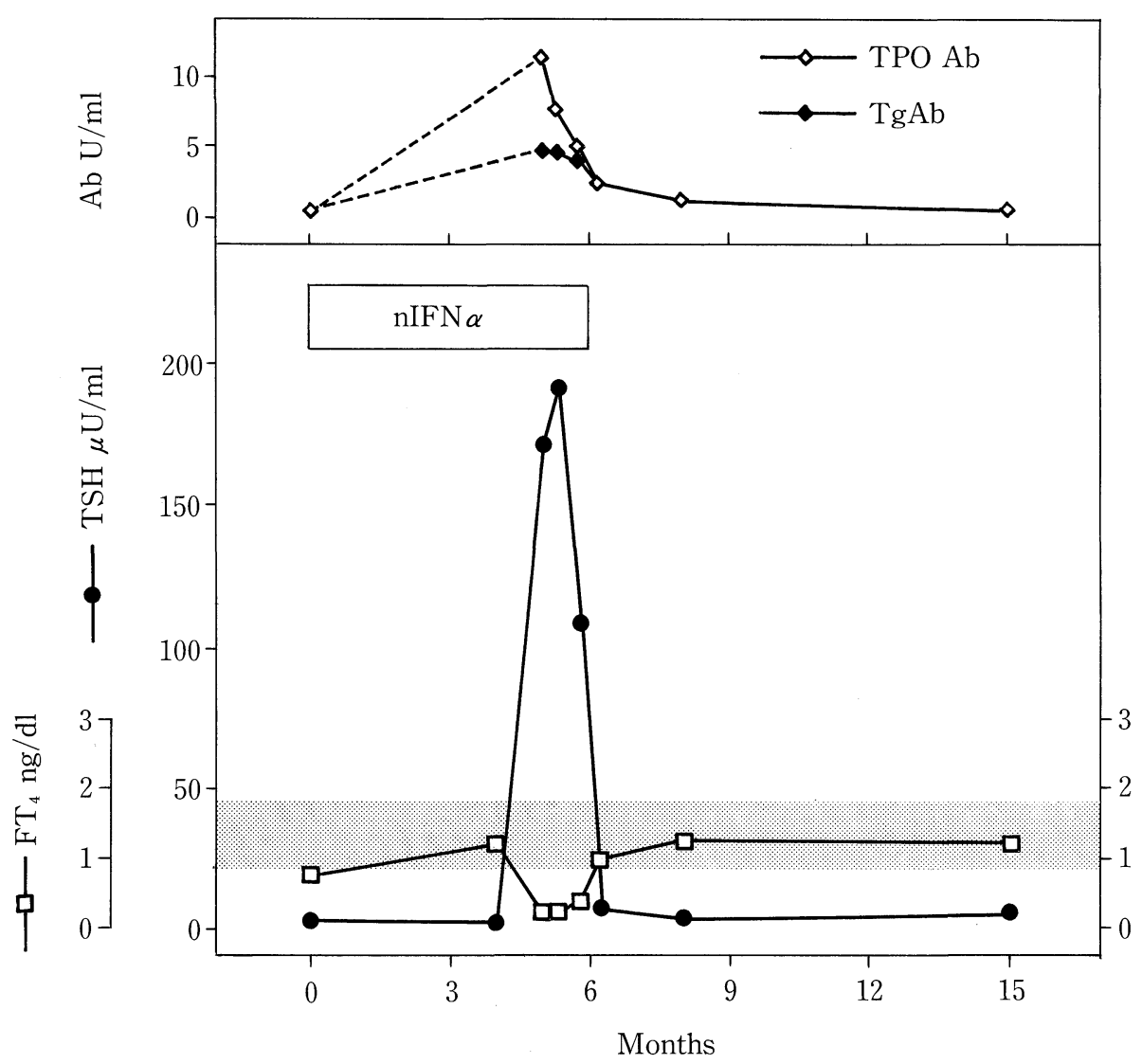

Figure 1. A 50-year-old female treated with nIFN $\alpha$, developed overt hypothyroidism. Shaded area indicates normal range of $\mathrm{FT}_{4}$.

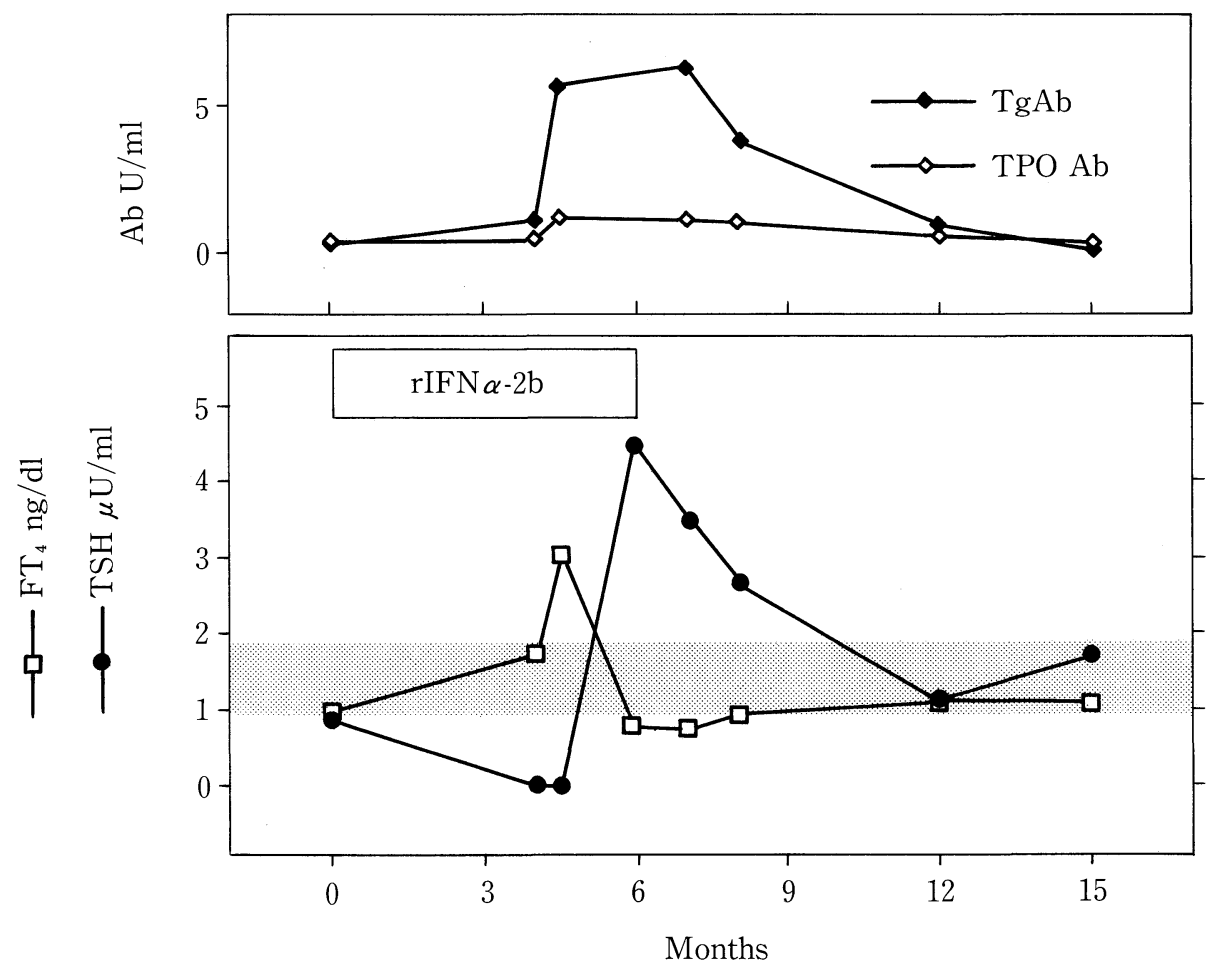

Figure 2. A 42-year-old female treated with rIFN $\alpha$-2b, developed transient thyrotoxicosis. Shaded area indicates normal range of $\mathrm{FT}_{4}$. 


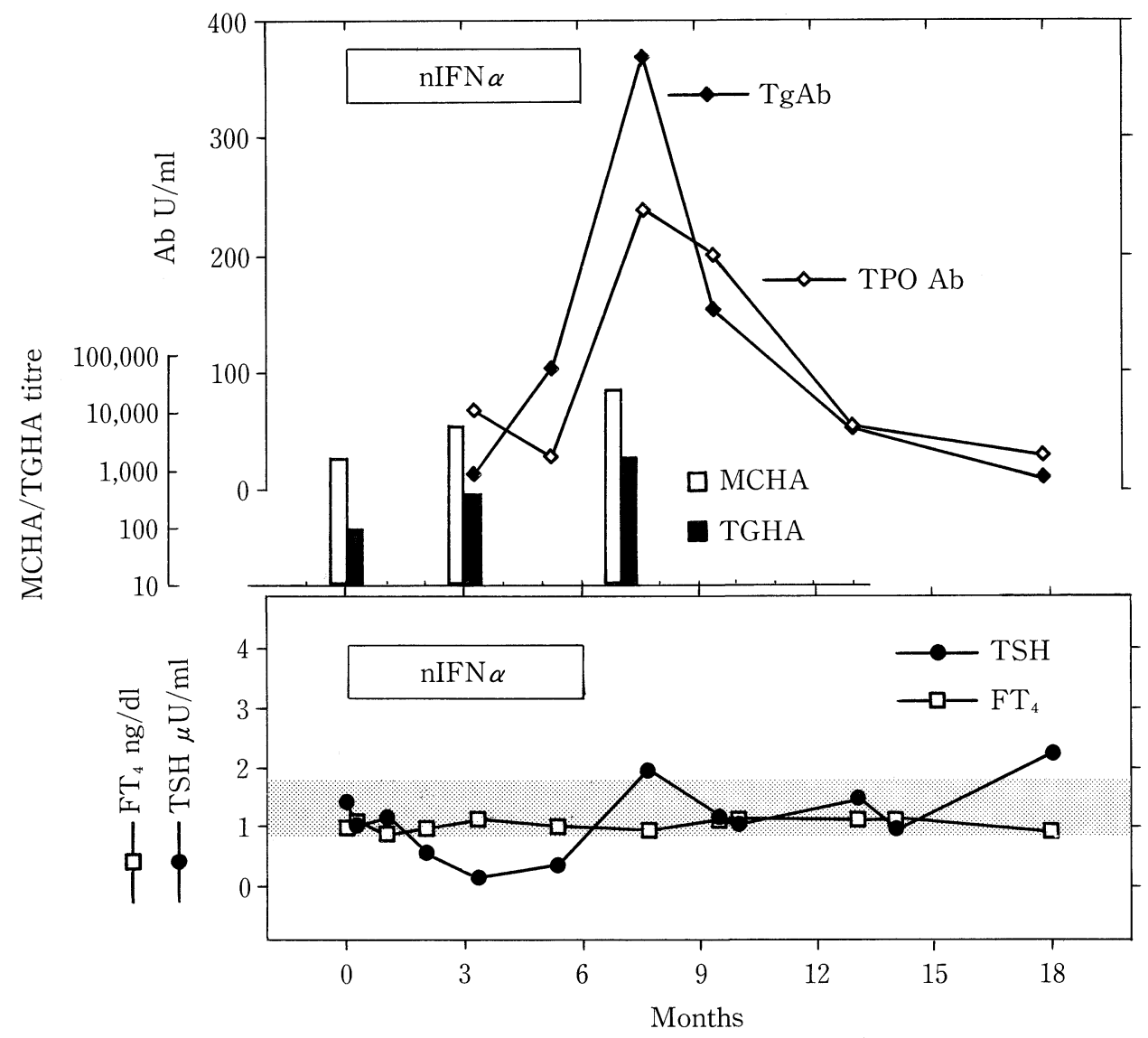

Figure 3. A 52-year-old female treated with nIFN $\alpha$, had no thyroid dysfunction but showed significant elevation of thyroid autoantibodies. Shaded area indicates normal range of $\mathrm{FT}_{4}$.

The patient completed IFN $\alpha$ therapy. The thyrotoxicosis was transient, the subsequent hypothyroidism was subclinical. $\mathrm{TPOAb}$ and $\mathrm{TgAb}$ were initially less than $0.1 \mathrm{U} / \mathrm{ml}$, and rose during treatment, but did not reach significant levels for HA. The elevation of thyroid autoantibodies was also transient.

$\mathrm{TgAb}$ and TPOAb also transiently elevated during and after IFN $\alpha$ therapy. TgAb was positive in $60.7 \%$ of the patients treated and TPOAb was positive in $53.3 \%$. Either $\mathrm{TgAb} / \mathrm{TPOAb}$ or both was positive in $71.9 \%$ of the patients treated. Figure 3 shows the clinical course in a 52-year-old female treated with nIFN $\alpha$, who developed marked elevation of thyroid autoantibodies. She was euthyroid with medium-sized and elastic goiter and had positive MCHA/TGHA before therapy. The thyroid function did not change but the autoantibodies rose during the therapy. The MCHA titer reached 25,600 at seven weeks after completion of IFN therapy. Five patients who developed thyroid dysfunction had positive MCHA before the treatment, but the remaining 14 patients had negative MCHA/ TGHA.

A total of 76 patients completed the 24-week therapy, 51 subjects responded to the therapy and 25 subjects did not. TSH was suppressed and/or elevated in 23 responders and 5 nonresponders. TSH changes significantly occurred in responders by chi-square analysis. $\mathrm{TgAb} / \mathrm{TPOAb}$ changes were recog- nized in a total of 18 out of 49 responders, while they were only recognized in 6 non-responders.

\section{Discussion}

We confirmed the IFN $\alpha$-induced thyroid abnormality is transient and reversible (4-6, 9-14). The signs and symptoms of thyrotoxicosis were subtle and sometimes difficult to detect in this study. The subclinical thyroid dysfunction detected by TSH, shown in the Fig. 2 may be overlooked, unless the thyroid function was determined at monthly intervals. Patients with chronic hepatitis have fatigue and discomfort before IFN therapy, and the therapy is also accompanied by acute side effects, such as fever, chills, myalgias, headache, fatigue or irritability in the early phase. Therefore, the symptoms of thyroid dysfunction may not be severe for the patients. Usually, thyroid abnormalities were reversed when IFN therapy was discontinued $(1,6)$. Some authors recommend discontinuation of IFN $\alpha$ therapy because of thyroid dysfunction. However, the thyroid dysfunction in our patients was transient without discontinuation, and normalized without L-thyroxine. Our data suggest that it is not necessary to discontinue the therapy when thyroid abnormality develops. Even in the event of severe hypothyroidism, we could continue the therapy with informed consent with or without 
supplementation of L-thyroxine. We (17) and other authors (6, 18) have experienced that patients with severe hypothyroidism induced by IFN $\alpha$ could discontinue the supplementation of Lthyroxine after one or two years.

IFN $\alpha$, in addition to its antiviral and anti-proliferative activities, acts as an immunomodulatory agent, including autoantibody production and development of autoimmune disease in susceptible patients $(4,5,9-11)$. The high prevalence of abnormal thyroid function and thyroid autoantibodies measured by sensitive assay in patients with chronic hepatitis $\mathrm{C}$ before IFN $\alpha$ therapy has recently been described $(2,3)$. In this study, we showed that the prevalence of goiter was high, and that in the majority of cases, the goiter was elastic and hard as in chronic thyroiditis. Elevated TSH, palpation and US findings of the thyroid suggest pre-existent autoimmune thyroid disease. It is well known that postpartum thyroid dysfunction occurs during the course of an occult chronic thyroiditis. Immunomodulatory events such as delivery can modify the natural history of Hashimoto's thyroiditis. Our data suggest that some patients have an autoimmune chronic thyroiditis prior to the therapy, and with IFN $\alpha$ treatment, the occult autoimmune disorder becomes overt. The occurrence of hypothyroidism in patients with thyroid autoantibodies is not unexpected, but we could not predict the development of thyroid dysfunction by MCHA/ TGHA, which was not measured by a sensitive assay such as the RIA before treatment. TPOAb and TgAb might be sensitive enough to detect IFN-induced immunomodulatory effect.

Postpartum thyroid dysfunction may be due to the transient rebound of the autoimmune process following cessation of pregnancy (19). In an earlier study (17), we reported patients with hypothyroidism and an elevated MCHA titer $(400-6,400)$ after their IFN $\alpha$ therapy had been completed. We speculated that stopping IFN $\alpha$ was a trigger for thyroid destruction, and we expected the thyroid dysfunction would occur after completion of IFN $\alpha$ therapy. In this study, TSH suppression/elevation or both was recognized before completion of the therapy, particularly in the latter half of the therapy, indicating that the thyroid dysfunction did not rise from the withdrawal mechanism.

Thyroid dysfunction was significantly more frequent in patients with IFN $\alpha$ therapy than in those without IFN $\alpha$ therapy. Thyroid dysfunction indicates an aspect of immunomodulatory action and may also indicate antiviral and antiproliferative activities. Some authors have suggested a positive relationship between the appearance of thyroid dysfunction and the response to IFN $\alpha$ therapy $(7,8,13)$. From the present results, IFNo-associated thyroid abnormalities in patients with chronic active hepatitis $\mathrm{C}$ represent a promising factor for beneficial response to therapy.

Acknowledgements: We would like to thank Robert E. Brandt for his invaluable assistance, proofreading and editing the manuscript.

\section{References}

1) Marazuela M, García-Buey L, Gonzáles-Fernández B, et al. Thyroid autoimmune disorders in patients with chronic hepatitis $\mathrm{C}$ before and during interferon- $\alpha$ therapy. Clin Endocrinol (Oxf) 44: 635, 1996.

2) Imagawa $\mathrm{A}$, Itoh $\mathrm{N}$, Hanafusa $\mathrm{T}$, et al. Autoimmune endocrine disease induced by recombinant interferon- $\alpha$ therapy for chronic active type $\mathrm{C}$ hepatitis. J Clin Endocrinol Metab 80: 922, 1995.

3) Tran A, Quaranta JF, Benzaken S, et al. High prevalence of thyroid autoantibodies in a prospective series of patients with chronic hepatitis $\mathrm{C}$ before interferon therapy. Hepatology 18: 253, 1993.

4) Nagayama $Y$, Ohta $K$, Tsuruta $M$, et al. Exacerbation of thyroid autoimmunity by interferon a treatment in patients with chronic viral hepatitis: our studies and review of the literature. Endocr J 41: 565, 1994.

5) Katabami S, Kamijo K, Kodama T, Fujisawa Y, Katanuma A, Yachi A. An episode of silent thyroiditis in a patient with chronic thyroiditis and papillary adenocarcinoma following alpha interferon treatment for hepatitis C. Endocrin J 40: 311, 1993.

6) Baudin E, Marcellin P, Pouteau M, et al. Reversibility of thyroid dysfunction induced by recombinant alpha interpheron in chronic hepatitis $C$. Clin Endocrinol (Oxf) 39: 657, 1993.

7) Primo J, Hinojosu J, Moles JR, et al. Development of thyroid dysfunction after $\alpha$-interferon treatment of chronic hepatitis C. Am J Gastroenterol 88: 1976, 1993 (letter; comment).

8) Lisker-Melman M, Di Bisceglie AM, Usala SJ, Weintraub B, Murray LM, Hoofnagle JH. Development of thyroid disease during therapy of chronic viral hepatitis with interferon alfa. Gastroenterology 102: 2155, 1992.

9) Chung YH, Shong YK. Development of thyroid autoimmunity after administration of recombinant human interferon- $\alpha 2 b$ for chronic viral hepatitis. Am J Gastroenterol 88: 244, 1993.

10) Gisslinger H, Gilly B, Woloszczuk W, et al. Thyroid autoimmunity and hypothyroidism during long-term treatment with recombinant interferonalpha. Clin Exp Immunol 90: 363, 1992.

11) Rönnblom LE, Alm GV, Öberg KE. Autoimmunity after alpha-interferon therapy for malignant carcinoid tumors. Ann Intern Med 115: 178, 1991.

12) Sauter NP, Atkins MB, Mier JW, Lechan RM. Transient thyrotoxicosis and persistent hypothyroidism due to acute autoimmune thyroiditis after interleukin- 2 and interferon- $\alpha$ therapy for metastatic carcinoma: a case report. Am J Med 92: 441, 1992.

13) Reid I, Sharpe I, McDevitt J, et al. Thyroid dysfunction can predict response to immunotherapy with interleukin-2 and interferon $\alpha-2 \mathrm{a}$. Br J Cancer 64: 915, 1991.

14) Pichert G, Jost LM, Zöbeli L, Odermatt B, Pedio G, Stahel RA. Thyroiditis after treatment with interleukin-2 and interferon $\alpha-2 \mathrm{a}$. Br J Cancer 62: 100, 1990.

15) Burman P, Tötterman TH, Öberg K, Karlsson FA. Thyroid autoimmunity in patients on long term therapy with leukocyte derived interferon. J Clin Endocrinol Metab 63: 1086, 1986.

16) Schultz M, Müller R, von zur Mühlen A, Brabant G. Induction of hyperthyroidism by interferon- $\alpha-2 b$. Lancet 1: 1452, 1989 (letter).

17) Tsuboi K, Matoba H, Yuasa R, Ihara F, Miyachi Y. Autoimmune thyroid disease in concealment-revealed by IFN $\alpha$. J Endocrinol Invest 17 (Supple, 1 to no 6): 61, 1994.

18) Sato K, Miyakawa M, Demura H. Reversible, extremely severe hypothyroidism in a patient with chronic hepatitis $C$ treated with interferon- $\alpha$. Thyroid 6: 249, 1996.

19) Amino N, Mori $\mathrm{H}$, Iwatani $\mathrm{Y}$, et al. High prevalence of transient postpartum thyrotoxicosis and hypothyroidism. NEngl J Med 306: 849, 1982. 